\title{
ZeroCostDL4Mic: an open platform to use Deep-Learning in Microscopy
}

\author{
Lucas von Chamier $^{1 *}$, Romain F. Laine ${ }^{1,2 *}$, Johanna Jukkala ${ }^{3,4}$, Christoph Spahn $^{5}$, Daniel Krentzel ${ }^{6,7}$, Elias Nehme ${ }^{8,9}$, \\ Martina Lerche ${ }^{3}$, Sara Hernández-Pérez ${ }^{3,10}$, Pieta K. Mattila ${ }^{3,10}$, Eleni Karinou ${ }^{11}$, Séamus Holden ${ }^{11}$, Ahmet Can Solak ${ }^{12}$, \\ Alexander Krull $^{13-15}$, Tim-Oliver Buchholz ${ }^{13,14}$, Martin L. Jones ${ }^{6}$, Loïc A Royer ${ }^{12}$, Christophe Leterrier ${ }^{16}$, Yoav Shechtman ${ }^{9}$, \\ Florian Jug $^{13,14,17}$, Mike Heilemann ${ }^{5}$, Guillaume Jacquemet ${ }^{3,4 凶}$, and Ricardo Henriques ${ }^{1,2,18 \bowtie}$
}

\author{
${ }^{1}$ MRC-Laboratory for Molecular Cell Biology, University College London, London, UK \\ ${ }^{2}$ The Francis Crick Institute, London, UK \\ ${ }^{3}$ Turku Bioscience Centre, University of Turku and Åbo Akademi University, Turku, Finland \\ ${ }^{4}$ Faculty of Science and Engineering, Cell Biology, Åbo Akademi University, Turku, Finland \\ ${ }^{5}$ Institute of Physical and Theoretical Chemistry, Goethe-University Frankfurt, Frankfurt, Germany \\ ${ }^{6}$ Electron Microscopy Science Technology Platform, The Francis Crick Institute, 1 Midland Road, London NW1 1AT, UK \\ ${ }^{7}$ Department of Bioengineering, Imperial College London, South Kensington Campus, London, SW7 2AZ, UK \\ ${ }^{8}$ Department of Electrical Engineering, Technion - Israel Institute of Technology, Haifa, Israel \\ ${ }^{9}$ Department of Biomedical Engineering, Technion - Israel Institute of Technology, Haifa, Israel \\ ${ }^{10}$ Institute of Biomedicine, and MediCity Research Laboratories, University of Turku, Finland \\ ${ }^{11}$ Centre for Bacterial Cell Biology, Biosciences Institute, Faculty of Medical Sciences, Newcastle University, UK \\ ${ }^{12}$ Chan Zuckerberg Biohub, San Francisco, CA, USA \\ ${ }^{13}$ Center for Systems Biology Dresden (CSBD), Dresden, Germany \\ ${ }^{14}$ Max Planck Institute for Molecular Cell Biology and Genetics, Dresden, Germany \\ ${ }^{15}$ Max Planck Institute for Physics of Complex Systems, Dresden, Germany \\ ${ }^{16}$ Aix Marseille Universite, CNRS, INP UMR7051, NeuroCyto, Marseille, France \\ ${ }^{17}$ Fondatione Human Technopole, Milano, Italy \\ ${ }^{18}$ Instituto Gulbenkian de Ciência, Oeiras, Portugal \\ *Equal contributing authors
}

The resources and expertise needed to use Deep Learning (DL) in bioimaging remain significant barriers for most laboratories. We present ZeroCostDL4Mic, a platform simplifying access to DL by exploiting the free, cloud-based computational resources of Google Colab. ZeroCostDL4Mic allows researchers to train, evaluate, and apply key DL networks to perform tasks including segmentation, detection, denoising, restoration, resolution enhancement and image-to-image translation. We demonstrate the application of the platform to study multiple biological processes.

Microscopy | Deep Learning | Machine Learning | Google Colab | Python | Education | Segmentation | Denoising | Image Restoration | Artificial Labeling | Super-resolution microscopy | Object detection | Classification | Image-toimage translation

Correspondence:

guillaume.jacquemet@abo.fi and rjhenriques@igc.gulbenkian.pt

Despite the enthusiasm and innovations fuelled by DL technology, the need to access powerful and compatible resources, install multiple computational tools, and modify code to train neural networks all lead to a significant accessibility barrier that is difficult to cross by scientists without a strong background in computer science. This includes most biologists, clinicians, pathologists and microscopy users. To perform a specific bioimage analysis task, a typical DL pipeline requires users first to train a neural network with appropriate training data. Once trained, the DL network can be applied to analyze images that are similar to those used during training. Training is the crucial part of the DL pipeline as it will dictate the specificity and performance of the DL network $(1,2)$. However, it is also a challenging aspect of the process as it requires specialized knowledge and access to specialised computational resources. As a result, researchers may find it easier to use pre-trained networks available online to process their images. This approach may alleviate the

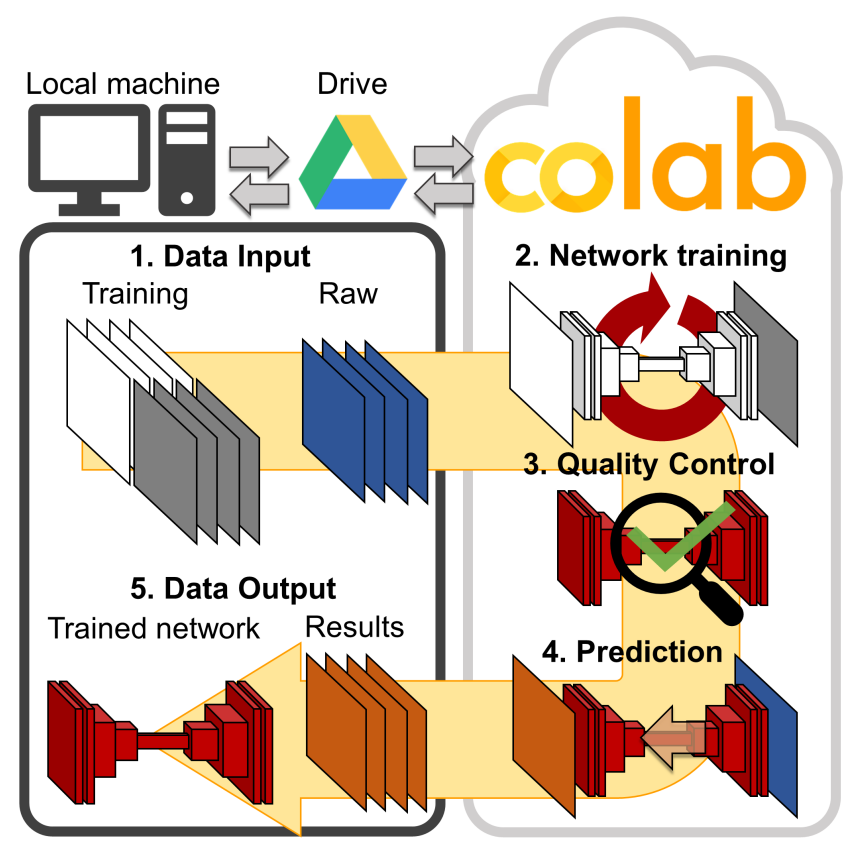

Fig. 1. Overview of ZeroCostDL4Mic. Workflow of ZeroCostDL4Mic, featuring data transfer through Google Drive, plus training, quality control and prediction via Google Colab. After running a network, both trained models and prediction results can then be downloaded to the user's machine.

onerous computational requirement of training and may even produce visually appealing imaging data. However, it has become clear that using pre-trained networks without appropriate additional training on the specific data of interest (a process known as transfer learning (3)), can lead to artifactual and misleading predictions (Supplementary Note 1).

Here, we present ZeroCostDL4Mic, an easy-to-use deploy- 

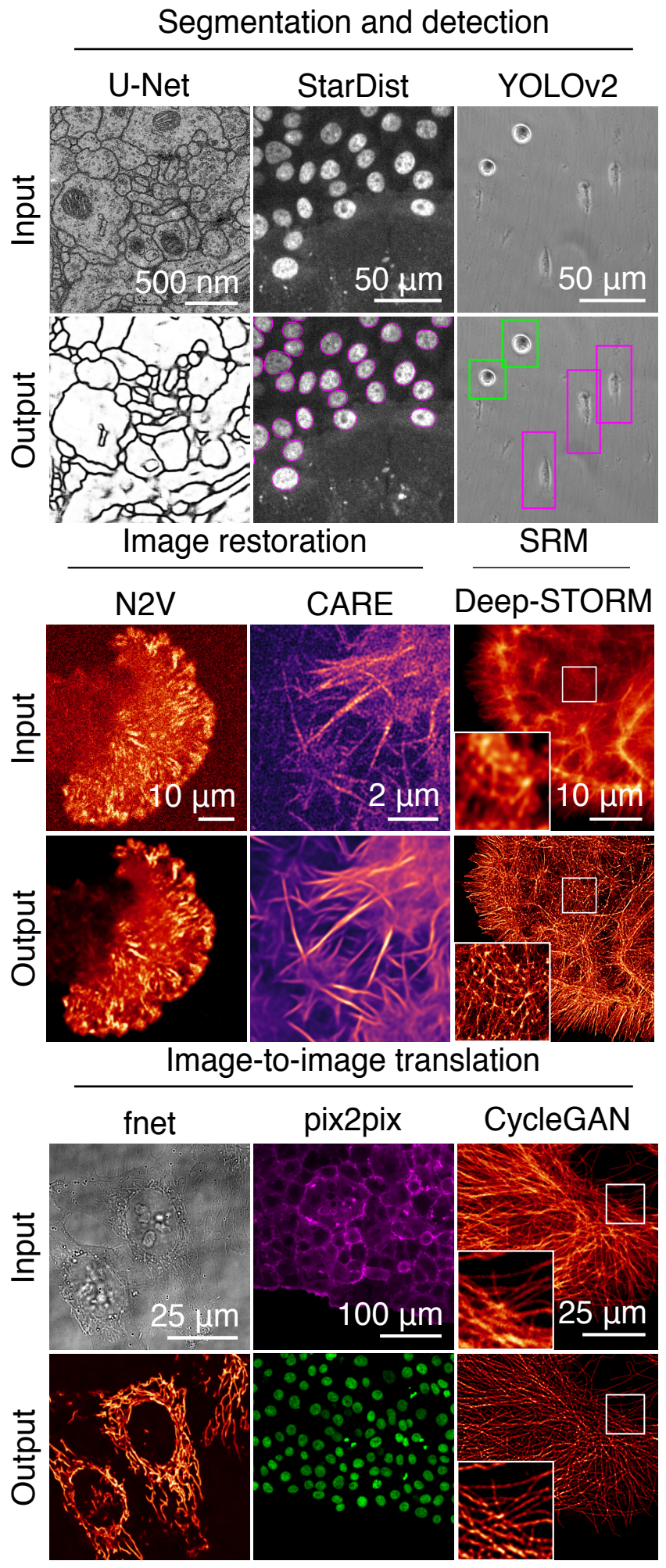

Fig. 2. Overview of the bioimage analysis tasks currently implemented within ZeroCostDL4Mic platform. Datasets from top left to bottom right: U-Net - ISBI 2012 Neuronal Segmentation Dataset16, Stardist - nuclear marker (SiR-DNA) in DCIS.COM cells, YOLOv2 - brightfield in MDA-MB-231 cells, N2V - actin label (paxillin-GFP) in U-251-glioma cells, CARE - actin label Lifeact-RFP in DCIS.COM cells, Deep-STORM - actin-labeled glial cell, fnet - brightfield and mitochondrial label TOM20-AlexaFluor 594 in HeLa cells, pix2pix - actin label Lifeact-RFP and nuclear labels in DCIS.COM cells, CycleGAN - tubulin label in U2OS cells. All datasets are available through Zenodo or as indicated in the GitHub repository.

ment DL platform which considerably simplifies the use of DL for microscopy (Supplementary Video 1). Importantly,
ZeroCostDL4Mic allows researchers with little or no coding expertise to train (and re-train), validate and use DL networks (Figure 1). In parallel, it guides researchers to acquire more knowledge, to experiment with optimizing DL parameters and generate the training data necessary for DL. It uses Google Colab which provides the free, cloud-based computational resources needed for each step in the DL pipeline. We currently offer solutions to multiple powerful bioimage analysis tasks made possible with DL within ZeroCostDL4Mic (Figure 2 and Supplementary Video 2). These include image segmentation and object detection (using UNet (4-6), StarDist $(7,8)$ and YOLOv2 (9)), image denoising and restoration (using CARE (10) and, Noise2Void (11)), super-resolution microscopy (using Deep-STORM (12)) and image-to-image translations (using Label-free prediction fnet (13), pix2pix (14) and CycleGAN (15)) (see Supplementary Note 2, Supplementary Fig. 2-7 and Supplementary Video 2-11).

In practice, ZeroCostDL4Mic is a collection of selfexplanatory Jupyter Notebooks, featuring an easy-to-use graphical user interface (GUI) (Supplementary Fig. 8) that requires only a web browser and a Google Drive account for a user to run any of our DL-based tasks. Jupyter Notebooks can efficiently and interactively run Python code, currently the default language to deploy DL applications. All calculations are performed in the cloud, circumventing the need to purchase or install graphical processing units (GPUs) and associated software.

Using ZeroCostDL4Mic does not require prior knowledge in coding. Researchers can, in a few mouse clicks and aided by a simple workflow, install all needed software dependencies, upload their imaging data and run networks for training and prediction (Supplementary Video 1). While the underlying code is hidden by default, it remains accessible, allowing users to learn, explore, and edit the notebooks' programmatic structure.

ZeroCostDL4Mic notebooks share a common workflow to promote easy adoption and good practice in DL, encouraging users to use models trained on their data. Indeed, before deploying trained models on unseen data, users can easily test their quality (see Supplementary Note 3 and Supplementary Fig. 9 and 10). This is fundamental in optimizing the network performance for a particular application, determining its limitations and preventing the significant introduction of artifacts, a commonly raised concern for DL applications in microscopy $(1,2)$. In practice, we implemented a quantitative quality control step in all notebooks, which allows the assessment (discussed in Supplementary Note 3) and improvement of model performance (Supplementary Fig. 11).

Additionally, we enabled several important functionalities that facilitate and improve the applicability of our DL approaches. In particular, we implemented: (1) automated data augmentation which can artificially expand the image diversity of a dataset, especially beneficial when only small training datasets are available (Supplementary Note 4 and Supplementary Fig. 12 and 13); (2) transfer learning (3), allowing to take advantage of pre-trained networks (from so-called model 
"zoos") by re-using previously learned features within these models and therefore speeding up and improving the training process (see Supplementary Note 5 and Supplementary Fig. 14 and 15); and (3) batch processing (predictions) of unseen data to streamline analysis once a satisfactory model has been obtained. It is important to note that trained models can also be downloaded and used outside ZeroCostDL4Mic (e.g., StarDist in Fiji(16)).

Via Google Colab, ZeroCostDL4Mic provides free access to the high-performance computational resources needed to run the large range of DL networks implemented here (Supplementary Note 2 for networks and Supplementary Note 6 for resources). For each featured network, we provide an example dataset that researchers can use to test and learn the basis of its workflow. For these datasets, we show that the corresponding training sessions only take a few minutes to a few hours (Supplementary Table 2), allowing the generation of high-performance DL models, producing the inference outputs shown in Figure 2 and the Supplementary Figures and Movies (Supplementary Fig. 2-6 and Supplementary Video $2-11$. We also highlight the versatility and power of ZeroCostDL4Mic by sequentially combining multiple DL tasks such as image-to-image translation and tracking, and by integrating it within larger image analysis pipelines to enable, for instance, automated cell tracking(17) (Supplementary Fig. 7). Therefore, we envision ZeroCostDL4Mic constitutes an easily accessible and adaptable starting point to the use and deployment of DL-based bioimage analysis.

ZeroCostDL4Mic complements current community efforts to simplify access to DL in microscopy, e.g., ImJoy (18) and ilastik (19) or integration projects of DL into Fiji/ImageJ $(7,8,10,16,20)$ it also substantially differs from these solutions by providing a single simple platform to carry out the necessary end-to-end DL workflow: install the various computational components, train a model using custom data, quantitatively validate the performance of the model and deployment on new data. By allowing training on custom data, we provide an alternative to the use of inappropriate pretrained models, which often do not correctly represent the types of data researchers will want to analyze (Supplementary Note 1 and Supplementary Fig. 1).

By bringing previously published methods into a streamlined format that allows easy, cost-free access and customized use of DL in microscopy, we believe this resource is an important step towards widening the use of DL approaches beyond the community of computer scientists to the laboratories that generate the imaging data. In parallel, it enables researchers to improve their understanding of DL and experiment with optimizing DL parameters and choosing appropriate networks for a specific application. These steps are essential to both exploit the benefits and understand the limitations of DL approaches in research. We envision that the templates presented here can be used by DL developers to showcase their own network architectures in a unified and reproducible framework (Supplementary Note 7). This will ensure the rapid dissemination of novel technologies and provide consistent user experience for reproducible and comparative studies of DL approaches. Altogether, ZeroCostDL4Mic has the potential to dramatically accelerate the uptake of DL for new users and promotes their capacity to use increasingly sophisticated and powerful imaging analysis strategies.

Availability. ZeroCostDL4Mic is available as Supplemental Software or can be accessed from our GitHub page. This resource is fully open-source, providing users with tutorials, Jupyter Notebooks for Google Colab, and many real-life example datasets for training and testing. The example datasets are available for download in Zenodo (links provided in Supplementary Table 1 and our GitHub page).

\section{ACKNOWLEDGEMENTS}

First and foremost, we would like to thank Dr. Martin Weigert from the Swiss Federal Institute of Technology (EPFL) and Dr. Uwe Schmidt from the Max Planck Institute of Molecular Cell Biology and Genetics (MPI-CBG), who pioneered a considerable portion of the technology this work is based on and whose ethos in making Deep Learning more accessible for microscopy helped inspire this work. Dr. Schmidt has kindly given key feedback during the preparation of the manuscript. All the network architectures and tasks presented here originate from already published work, having been edited and prepared for Google Colab to simplify their uptake by novice users. When using the ZeroCostDL4Mic platform, please cite the original publications associated with each network.

This work was funded by grants from the UK Medical Research Council (MR/K015826/1) (R.H.), the Wellcome Trust (203276/Z/16/Z) (R.H.) and the Gulbenkian Foundation (R.H.). R.F.L. would like to acknowledge the support of the MRC Skills development fellowship (MR/T027924/1). This work was also supported by grants awarded by the Academy of Finland (to G.J. and P.K.M.), the Sigrid Juselius Foundation (to G.J. and P.K.M.), the University of Turku foundation and Turku Doctoral Program in Molecular Medicine (TuDMM)(to SHP), Åbo Akademi University Research Foundation (CoE CellMech; G.J.) and by Drug Discovery and Diagnostics strategic funding to Åbo Akademi University (G.J.). We thank Dr. Aki Stubb for providing us with the raw data used to showcase Noise2Void 2D. The Cell Imaging and Cytometry Core facility (Turku Bioscience, University of Turku, Åbo Akademi University, and Biocenter Finland) and Turku Bioimaging are acknowledged for services and instrumentation and expertise. M.L is supported by Victoriastiftelsen (FI). A.K, T. - O.B and F.J. funded by German Research Foundation (DFG) under the code JU3110/1-1 and German Federal Ministry of Research and Education under the code 01IS18026C, ScaDS2. M.H. and C.S. acknowledge funding by the German Science Foundation (grant nr. SFB1177), C.S. additionally acknowledges support by the European Molecular Biology Organization (short term fellowship 8589). C.L. acknowledges the support of CNRS through the ATIP A0 2016 grant. SH and EK were supported by a Wellcome Trust Royal Society Sir Henry Dale Fellowship grant number $206670 / Z / 17 / Z$ to $S H$. We additionally thank Chan Zuckerberg Biohub and its donors for funding Loic A. Royer and Ahmet Can Solak's work. This work was supported by the Francis Crick Institute, which receives its core funding from Cancer Research UK (FC001999), the UK Medical Research Council (FC001999), and the Wellcome Trust (FC001999). Note: CoLaboratory ${ }^{\mathrm{TM}}$ and Google Drive ${ }^{\mathrm{TM}}$ are trademarks of Google LLC - (02018 Google LLC All rights reserved.

\section{EXTENDED AUTHOR INFORMATION}

- Lucas von Chamier: (D0000-0002-9243-912X; ChamierLucas

- Romain F. Laine: (D0000-0002-2151-4487; LaineBiolmaging

- Johanna Jukkala: $y$ Liehuletti

- Christoph Spahn: (D0000-0001-9886-2263; YmiCHRIScopy

- Daniel Krentzel: ${ }^{D}$ DanielKrentzel

- Martina Lerche: (100000-0002-8203-1120; Jmartina_lerche

- Sara Hernández-Pérez: (100000-0002-0227-1045; ysara_mattilalab

- Pieta K. Mattila: (D)0000-0003-2805-0686; Ymattilalab

- Eleni Karinou: (100000-0002-1099-4964; JKar_EI

- Seamus Holden: (1D0000-0002-7169-907X; $\mathbf{y}$ seamus_holden

- Ahmet Can Solak: (D0000-0002-1381-8309; ע_ahmetcansolak

- Alexander Krull: $y$ sagzehn

- Tim-Oliver Buchholz: (100000-0001-6953-8915; Ytibuch_

- Martin L. Jones: (D0000-0003-0994-5652; ymartinjones78

- Loïc A Royer: (100000-0002-9991-9724; Yloicaroyer

- Christophe Leterrier: (D)0000-0002-2957-2032; @christlet

- Yoav Shechtman: (D0000-0001-8498-5203; ShechtmanLab

- Florian Jug: (1D0000-0002-8499-5812; yflorianjug

- Mike Heilemann: (100000-0002-9821-3578

- Guillaume Jacquemet: (D)0000-0002-9286-920X; guijacquemet

- Ricardo Henriques: (D)0000-0002-2043-5234; yHenriquesLab

\section{AUTHOR CONTRIBUTIONS}

G.J. and R.H. conceived the project; L.v.C., R.F.L, J.J., D.K., E.N., T.-O.B. and G.J., and R.H. wrote source code based on the work of A.K., T.-O.B., F.J., E.N., Y.S., and L.A.R. among others; L.v.C., R.F.L., J.J., C.S., C.L., and G.J. performed the image 
bioRxiv preprint doi: https://doi.org/10.1101/2020.03.20.000133; this version posted August 17, 2020. The copyright holder for this preprint (which was not certified by peer review) is the author/funder, who has granted bioRxiv a license to display the preprint in perpetuity. It is made available under aCC-BY 4.0 International license.

acquisition of the test and example data; L.v.C., R.F.L., J.J., C.S., M.L., S.H.-P., P.K.M., E.K., S.H., A.K., T.-O. B., C.L., M.L.J., D.K., E.N., Y.S., M.H., and G.J. tested the platform; L.v.C., R.F.L., J.J., C.S., G.J. and R.H. wrote the manuscript with input from all co-authors.

\section{COMPETING FINANCIAL INTERESTS}

We provide a platform based on Google Drive and Google Colab to streamline the implementation of common Deep Learning analysis of microscopy data. Despite heavily relying on Google products, we have no commercial or financial interest in promoting and using them. In particular, we did not receive any compensation in any form from Google for this work. The authors declare no competing financial interests.

\section{Bibliography}

1. Chinmay Belthangady and Loic A Royer. Applications, promises, and pitfalls of deep learning for fluorescence image reconstruction. Nature methods, pages 1-11, 2019.

2. Lucas von Chamier, Romain $\mathrm{F}$ Laine, and Ricardo Henriques. Artificial intelligence for microscopy: what you should know. Biochemical Society Transactions, 47(4):1029-1040, 2019.

3. Sinno Jialin Pan and Qiang Yang. A survey on transfer learning. ieee transactions on knowledge and data engineering. 22 (10): 1345, 1359, 2010.

4. Olaf Ronneberger, Philipp Fischer, and Thomas Brox. U-net: Convolutional networks for biomedical image segmentation. In International Conference on Medical image computing and computer-assisted intervention, pages 234-241. Springer, 2015.

5. Özgün Çiçek, Ahmed Abdulkadir, Soeren S Lienkamp, Thomas Brox, and Olaf Ronneberger. 3d u-net: learning dense volumetric segmentation from sparse annotation. In International conference on medical image computing and computer-assisted intervention, pages 424-432. Springer, 2016.

6. Thorsten Falk, Dominic Mai, Robert Bensch, Özgün Çiçek, Ahmed Abdulkadir, Yassine Marrakchi, Anton Böhm, Jan Deubner, Zoe Jäckel, Katharina Seiwald, et al. U-net: deep learning for cell counting, detection, and morphometry. Nature methods, 16(1):67-70, 2019

7. Uwe Schmidt, Martin Weigert, Coleman Broaddus, and Gene Myers. Cell detection with star-convex polygons. In International Conference on Medical Image Computing and Computer-Assisted Intervention, pages 265-273. Springer, 2018.

8. Martin Weigert, Uwe Schmidt, Robert Haase, Ko Sugawara, and Gene Myers. Star-convex polyhedra for $3 \mathrm{~d}$ object detection and segmentation in microscopy. In The IEEE Winter Conference on Applications of Computer Vision, pages 3666-3673, 2020.

9. Joseph Redmon and Ali Farhadi. Yolo9000: better, faster, stronger. In Proceedings of the IEEE conference on computer vision and pattern recognition, pages 7263-7271, 2017.

10. Martin Weigert, Uwe Schmidt, Tobias Boothe, Andreas Müller, Alexandr Dibrov, Akanksha Jain, Benjamin Wilhelm, Deborah Schmidt, Coleman Broaddus, Siân Culley, et al. Contentaware image restoration: pushing the limits of fluorescence microscopy. Nature methods, 15(12):1090-1097, 2018.

11. Alexander Krull, Tim-Oliver Buchholz, and Florian Jug. Noise2void-learning denoising from single noisy images. In Proceedings of the IEEE Conference on Computer Vision and Pattern Recognition, pages 2129-2137, 2019.

12. Elias Nehme, Lucien E Weiss, Tomer Michaeli, and Yoav Shechtman. Deep-storm: superresolution single-molecule microscopy by deep learning. Optica, 5(4):458-464, 2018.

13. Chawin Ounkomol, Sharmishtaa Seshamani, Mary M Maleckar, Forrest Collman, and Gregory R Johnson. Label-free prediction of three-dimensional fluorescence images from transmitted-light microscopy. Nature methods, 15(11):917-920, 2018.

14. Phillip Isola, Jun-Yan Zhu, Tinghui Zhou, and Alexei A Efros. Image-to-image translation with conditional adversarial networks. In Proceedings of the IEEE conference on computer vision and pattern recognition, pages 1125-1134, 2017.

15. Jun-Yan Zhu, Taesung Park, Phillip Isola, and Alexei A Efros. Unpaired image-to-image translation using cycle-consistent adversarial networks. In Proceedings of the IEEE international conference on computer vision, pages 2223-2232, 2017.

16. Johannes Schindelin, Ignacio Arganda-Carreras, Erwin Frise, Verena Kaynig, Mark Longair, Tobias Pietzsch, Stephan Preibisch, Curtis Rueden, Stephan Saalfeld, Benjamin Schmid, et al. Fiji: an open-source platform for biological-image analysis. Nature methods, 9(7): 676-682, 2012.

17. Jean-Yves Tinevez, Nick Perry, Johannes Schindelin, Genevieve M Hoopes, Gregory D Reynolds, Emmanuel Laplantine, Sebastian Y Bednarek, Spencer L Shorte, and Kevin W Eliceiri. Trackmate: An open and extensible platform for single-particle tracking. Methods, 115:80-90, 2017.

18. Wei Ouyang, Florian Mueller, Martin Hjelmare, Emma Lundberg, and Christophe Zimmer. Imjoy: an open-source computational platform for the deep learning era. Nature methods, 16(12):1199-1200, 2019.

19. Stuart Berg, Dominik Kutra, Thorben Kroeger, Christoph N Straehle, Bernhard X Kausler, Carsten Haubold, Martin Schiegg, Janez Ales, Thorsten Beier, Markus Rudy, et al. Ilastik: interactive machine learning for (bio) image analysis. Nature Methods, pages 1-7, 2019.

20. Estibaliz Gómez-de Mariscal, Carlos García-López-de Haro, Laurène Donati, Michael Unser, Arrate Muñoz-Barrutia, and Daniel Sage. Deepimagej: A user-friendly plugin to run deep learning models in imagej. bioRxiv, page 799270, 2019. 